\title{
Development and validation of a nomogram for predicting survival in patients with non-metastatic primary adenocarcinoma of the bladder
}

\author{
Dong-Dong Yu ${ }^{1 \#}$, Hui Dong ${ }^{2 \#}$, Wei-Kang Chen ${ }^{1 \#}$, Kun Chen ${ }^{3}$, Zhi-Gang Wu ${ }^{1}$, Cheng-Di Li ${ }^{1}$, Jian $\mathrm{Cai}^{1}$, \\ Yun-Bei Xiao ${ }^{1}$ \\ ${ }^{1}$ Department of Andrology, ${ }^{2}$ Department of Respiratory Medicine, ${ }^{3}$ Department of Thoracic Surgery, The First Affiliated Hospital of Wenzhou \\ Medical University, Wenzhou, China \\ Contributions: (I) Conception and design: All authors; (II) Administrative support: YB Xiao, J Cai; (III) Provision of study materials or patients: \\ All authors; (IV) Collection and assembly of data: DD Yu, H Dong, WK Chen; (V) Data analysis and interpretation: All authors; (VI) Manuscript \\ writing: All authors; (VII) Final approval of manuscript: All authors. \\ \#These authors contributed equally to this work. \\ Correspondence to: Dr. Yun-Bei Xiao; Prof. Dr. Jian Cai. Department of Urology, The First Affiliated Hospital of Wenzhou Medical University, \\ Wenzhou 325000, China. Email: xiaoyunbei121@163.com; cjhospital@yeah.net.
}

Background: To develop a nomogram for predicting cancer-specific survival (CSS) of patients with nonmetastatic primary adenocarcinoma of the bladder (NMACB).

Methods: We used a retrospective cohort study design. Patient data were obtained from the SEER database, univariate and multivariate Cox regression analyses were performed to identify factors associated with CSS. A nomogram visualization model was established using $\mathrm{R}$ language software to predict survival rate. Harrell's concordance index (C-index), area under the receiver operating characteristic (ROC) curve (AUC) in addition to calibration plots were used to assess the performance of the model.

Results: A total of 1,635 patients were included in the study. A multivariate Cox regression model indicated that age, histological type, grade, stage, and surgery were independent covariates associated with CSS. Using these prognostic factors, a nomogram was constructed. Harrell's C indices for CSS were 0.729 in the training cohort and 0.716 in the validation cohort. AUC values were $0.769,0.735$ and 0.724 for 1,3 , and 5 -year in the training cohort, and $0.738,0.727$ and 0.713 for 1, 3 and 5-year in the validation cohort, respectively. The AUC values and calibration plots indicated that the nomogram provided good predictive performance.

Conclusions: A nomogram for predicting CSS in patients with NMACB was developed to assist clinicians in the accurate prediction of mortality risk to allow them to recommend a personalized treatment modality.

Keywords: Cancer-specific survival (CSS); nomogram; non-metastatic primary adenocarcinoma of the bladder (NMACB); Surveillance, Epidemiology, and End Results (SEER)

Submitted Jan 09, 2020. Accepted for publication Jul 27, 2020.

doi: $10.21037 /$ tcr-20-354

View this article at: http://dx.doi.org/10.21037/tcr-20-354

\section{Introduction}

Bladder cancer is the most common cancer of the urinary system (1), of which greater than $90 \%$ of pathological types are urothelial carcinoma (2). Primary adenocarcinoma of the bladder (ACB) is a rare form that has a high degree of malignancy, late staging, and poor prognosis (3), accounting for approximately $0.5-2.0 \%$ of all bladder malignant tumors. ACB is classified as a primary non-urachal or urachal adenocarcinoma (4). Schistosomiasis (5) and bladder exstrophy (6) are thought to be associated with ACB. Bladder urothelial carcinoma has been widely investigated (7-9), while conversely, ACB has been rarely studied due to 
its low incidence rate.

Accurate estimates of prognosis based on clinicopathological factors play an important role in the selection of treatment strategies (10). Nomograms have been widely used to estimate a specific end point in cancer patients because they can improve relative predictive accuracy $(11,12)$. Multiple nomograms have been established to inform clinical practice in bladder cancer (13-15). The majority of nomograms focus on urothelial carcinoma of the bladder. There are significant differences in etiology, treatment, and prognosis between primary bladder adenocarcinoma and urothelial cancer. Nomograms developed for use with bladder urothelial carcinoma are not suitable for patients with ACB. However no predictive nomograms have been developed for ACB due to its low incidence rate. Prognosis and therapeutic options for nonmetastatic versus metastatic ACB vary widely. The present study focused on non-metastatic primary adenocarcinoma of the bladder (NMACB).

Here, we developed and validated a nomogram to predict survival in patients with NMACB from data in the surveillance, epidemiology and end results (SEER) database. The present study aimed at the identification of independent prognostic factors in patients with NMACB and also predict their cancer-specific survival (CSS). We present the following article in accordance with the STROBE reporting checklist (available at http://dx.doi. org/10.21037/tcr-20-354).

\section{Methods}

We used a retrospective cohort study design. Patient data were obtained from the National Cancer Institute's SEER program, which encompasses approximately $28 \%$ of the U.S. population. We used the International Statistical Classifications of Diseases for Oncology, 3rd edition (ICD-O-3) site codes C670-C679 and histology codes $8140-8147$ and $8255-8490$ to identify primary bladder tumors with adenocarcinoma. Additional inclusion criteria included: (I) bladder adenocarcinoma that was the first malignancy; (II) cancer at a non-metastatic tumor stage; (III) patients for whom information about CSS, duration of survival (in months) and therapy provided, were available; (IV) diagnosis by histological confirmation only. Cases diagnosed by clinical presentation, radiography or autopsy alone were excluded. This study was conducted in accordance with standard guidelines and was approved by the local Ethics Committee. We also got permission from the National Cancer Institute USA to access the SEER dataset for research purposes only (reference number: 18015-Nov2017). All the data from the SEER database were de-identified, and the extracted data did not require informed consent.

\section{Statistical analysis}

Patients were randomly assigned to either the training or validation cohort. Continuous variables such as age are presented as medians and the median value of the interquartile range (IQR). Categorical variables such as race are presented as counts and percentages. Univariate analysis was used to identify potential risk factors. Multivariate analyses were performed after the risk factors were identified. A statistical significance level of 0.05 was used to select variables for the nomogram. After comparing three different models (full model, stepwise model, multivariable fractional polynomial model), the full model displayed the best fit. A nomogram was developed based on a multivariate model derived from a Cox regression model. Harrell's concordance index (C-index) and area under the receiver operating characteristic (ROC) curve (AUC), in addition to calibration plots, were used to assess the performance of the model. Data were analyzed with the use of the statistical package R ( $\mathrm{R}$ foundation; http://www.r-project.org; version 3.4.3) and EmpowerStats software (www.empowerstats.com, $\mathrm{X} \& \mathrm{Y}$ solutions, Inc. Boston MA).

\section{Results}

\section{Demographic characteristics}

A total of 1,635 patients that satisfied the inclusion criteria described above were included in the analysis (Figure 1). The demographic and clinicopathological characteristics of the study patients are presented in Table 1. Median age was 66 years of age (range, 17 to 101 years). The majority of patients, $1,437(87.89 \%)$ suffered non-urachal primary adenocarcinomas of the bladder, 1,312 (80.24\%) were white and 1,050 (64.22\%) were male. Median followup time was 38 months (range, 1 to 403 months). A total of $1,120(68.50 \%)$ patients had died prior to the final follow-up, of which 715 (43.73\%) had died due to primary adenocarcinoma of the bladder (ACB). There were no significant differences of the variables between the training and validation cohorts. 


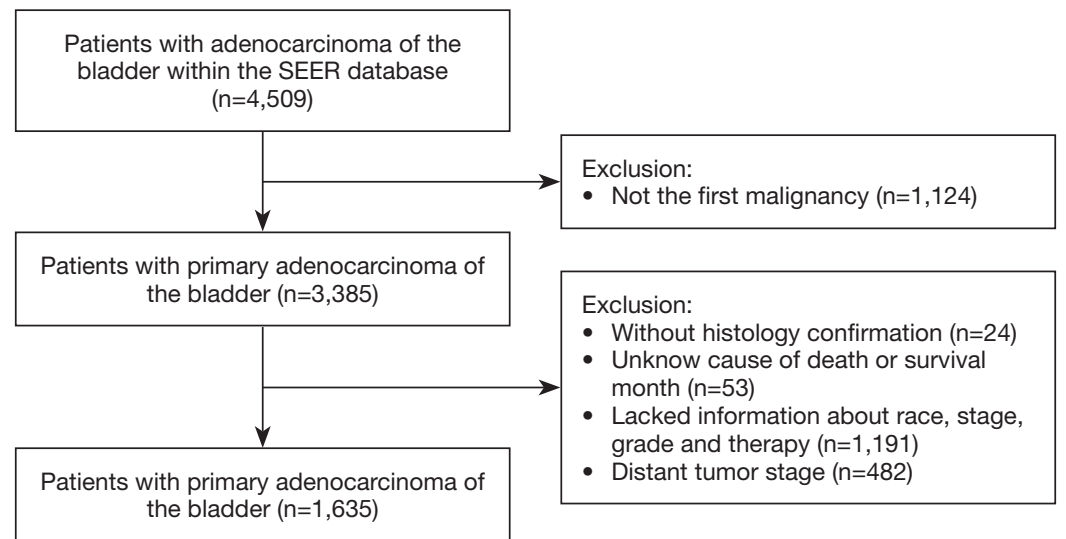

Figure 1 Flow-chart of the participants' selection.

\section{Pathologic and clinical characteristics}

When referring to stage and grade, the majority of patients, $1,156(70.70 \%)$ were of regional tumor stage and 698 (42.69\%) had poorly differentiated (Grade III) tumors. A total of $1,585(96.94 \%)$ patients underwent cancer-directed surgery. Of these, $616(37.68 \%)$ underwent transurethral surgery, $423(25.87 \%)$ received partial cystectomy and 491 (30.03\%) had complete cystectomy. In patients with a urachal primary site, most patients received either partial $(151,76.26 \%)$ or complete cystectomy $(25,12.63 \%)$. A small proportion of patients received chemotherapy (326, $19.94 \%)$ or radiation treatment $(182,11.13 \%)$.

\section{Survival and mortality analyses}

Univariate Cox analysis of the training cohort $(\mathrm{n}=1,226)$ (Table 2) indicated that worse prognosis was associated with older age (HR: 1.02, $\mathrm{P}<0.0001)$, being moderately differentiated (HR: 1.97, $\mathrm{P}=0.0016$ ), poorly differentiated (HR: 3.64, $\mathrm{P}<0.0001$ ), undifferentiated or anaplastic (HR: 2.90, $\mathrm{P}<0.0001$ ), or with a regional stage (HR: 2.13, $\mathrm{P}<0.0001)$.

Using adenocarcinoma not otherwise specified (NOS) as a reference, signet cell carcinoma (HR: $2.12, \mathrm{P}<0.0001)$ was shown to confer the worst survival outcome of the various histological subtypes while mucinous adenocarcinoma (HR: $0.95, \mathrm{P}=0.6277$ ) and other adenocarcinoma subtypes (HR: $0.98, \mathrm{P}=0.8662$ ) conferred similar survival outcomes.

Multivariate cox regression analysis (Table 3) demonstrated that worse prognosis was related to older age (HR: 1.02, $\mathrm{P}<0.0001$ ), being moderately differentiated (HR: $1.86, \mathrm{P}=0.0047$ ), poorly differentiated (HR: $2.53, \mathrm{P}<0.0001$ ), undifferentiated or anaplastic (HR: 2.01, $\mathrm{P}=0.0035)$, a tumor that is at a regional stage (HR: $2.59, \mathrm{P}<0.0001)$ or is a mucinous adenocarcinoma (HR: $1.36, \mathrm{P}=0.0138$ ). However, being female (HR: 1.17, $\mathrm{P}=0.0905$ ) or having a non-urachal primary site (HR: $1.27, \mathrm{P}=0.1839$ ) were statistically insignificant in the multivariate cox regression model. Signet cell carcinoma (HR: $1.80, \mathrm{P}=0.0009$ ) was also shown to represent a survival disadvantage compared with adenocarcinoma NOS. Patients who had received surgery (HR: $0.38, \mathrm{P}<0.0001$ for transurethral surgery; HR: 0.15, $\mathrm{P}<0.0001$ for partial cystectomy; HR: $0.24, \mathrm{P}<0.0001$ for cystectomy; HR: $0.33, \mathrm{P}=0.0006$ for other forms of surgery) had a better prognosis than those who had not, while radiotherapy (HR: 1.07, $\mathrm{P}=0.6124$ ) and chemotherapy (HR: $1.20, \mathrm{P}=0.1139$ ) became statistically insignificant which was due mostly to tumor stage (exclusion of tumor stage in the complete model had the greatest influence on the regression coefficient for radiotherapy and chemotherapy, data not shown in the article).

\section{Development and validation of the nomogram}

Univariate analysis was used to identify potential risk factors. Independent prognostic factors were selected from the results of multivariate cox analyses. A statistical significance level of 0.05 was used to select the variables in multivariate analyses to develop a nomogram for predicting the 1,3 and 5-year CSS of ACB patients. A total of 1,635 patients were randomly assigned to the training $(n=1,226)$ and validation cohort $(n=409)$ cohorts. The nomogram for predicting 1, 3 and 5-year CSS in patients with ACB is presented in Figure 2. 
Table 1 Clinical characteristics of the 1,635 patients with primary adenocarcinoma of the bladder

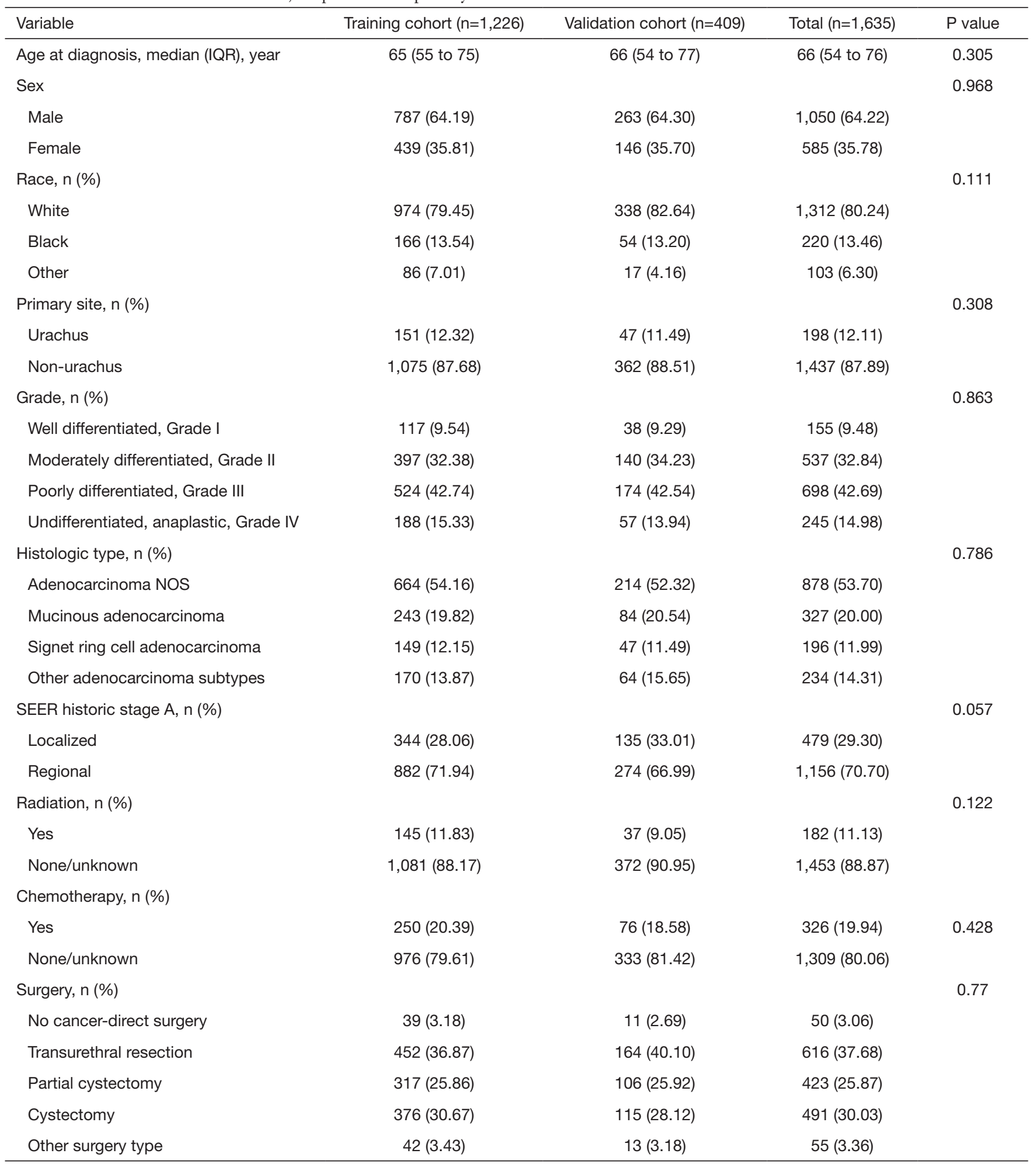

Localized, confined entirely to the organ of origin. Regional, has extended (I) beyond the limits of the organ of origin directly into surrounding organs or tissues; (II) into regional lymph nodes by way of the lymphatic system; or (III) by a combination of extension and regional lymph nodes. Distant, has spread to parts of the body remote from the primary tumor either by direct extension or by discontinuous metastasis. IQR, interquartile range. 
Table 2 Univariate Cox regression analysis of prognostic factors for cancer specific survival in ACB

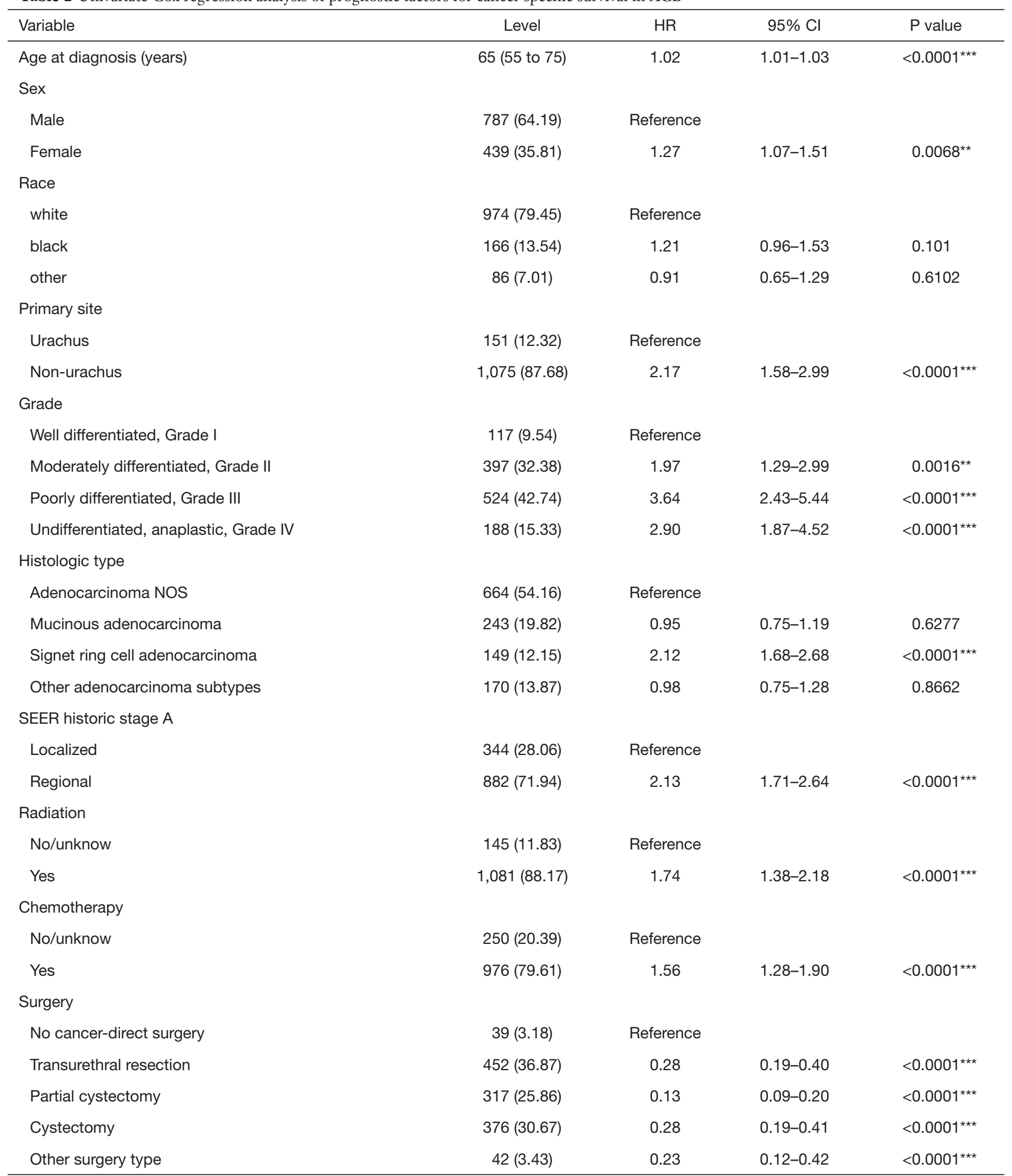

Data are presented as median (IQR) or $\mathrm{n}(\%) .{ }^{* \star}, \mathrm{P}<0.01$; ${ }^{* \star}, \mathrm{P}<0.001$. ACB, adenocarcinoma of the bladder; $\mathrm{Cl}$, confidence interval; HR, hazard ratio. 
Table 3 Multivariate Cox regression analysis of prognostic factors for cancer specific survival in ACB

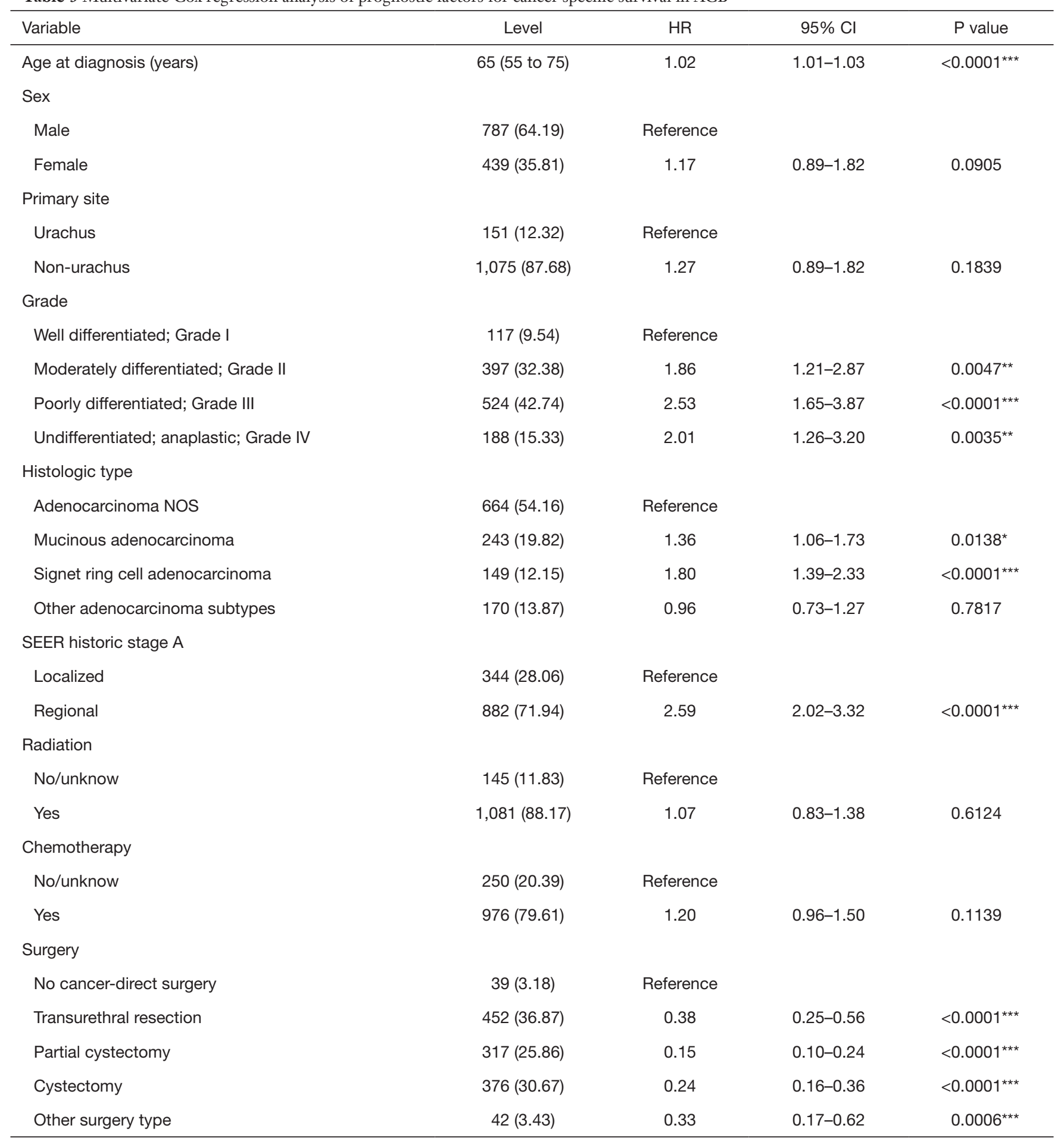

Data are presented as median (IQR) or $\mathrm{n}(\%) .{ }^{*}, \mathrm{P}<0.05 ;{ }^{* \star}, \mathrm{P}<0.01 ;{ }^{* *}, \mathrm{P}<0.001$. Multivariable Cox regression hazards models were also adjusted for diagnosis year and race. $\mathrm{ACB}$, adenocarcinoma of the bladder; $\mathrm{Cl}$, confidence interval; HR, hazard ratio. 

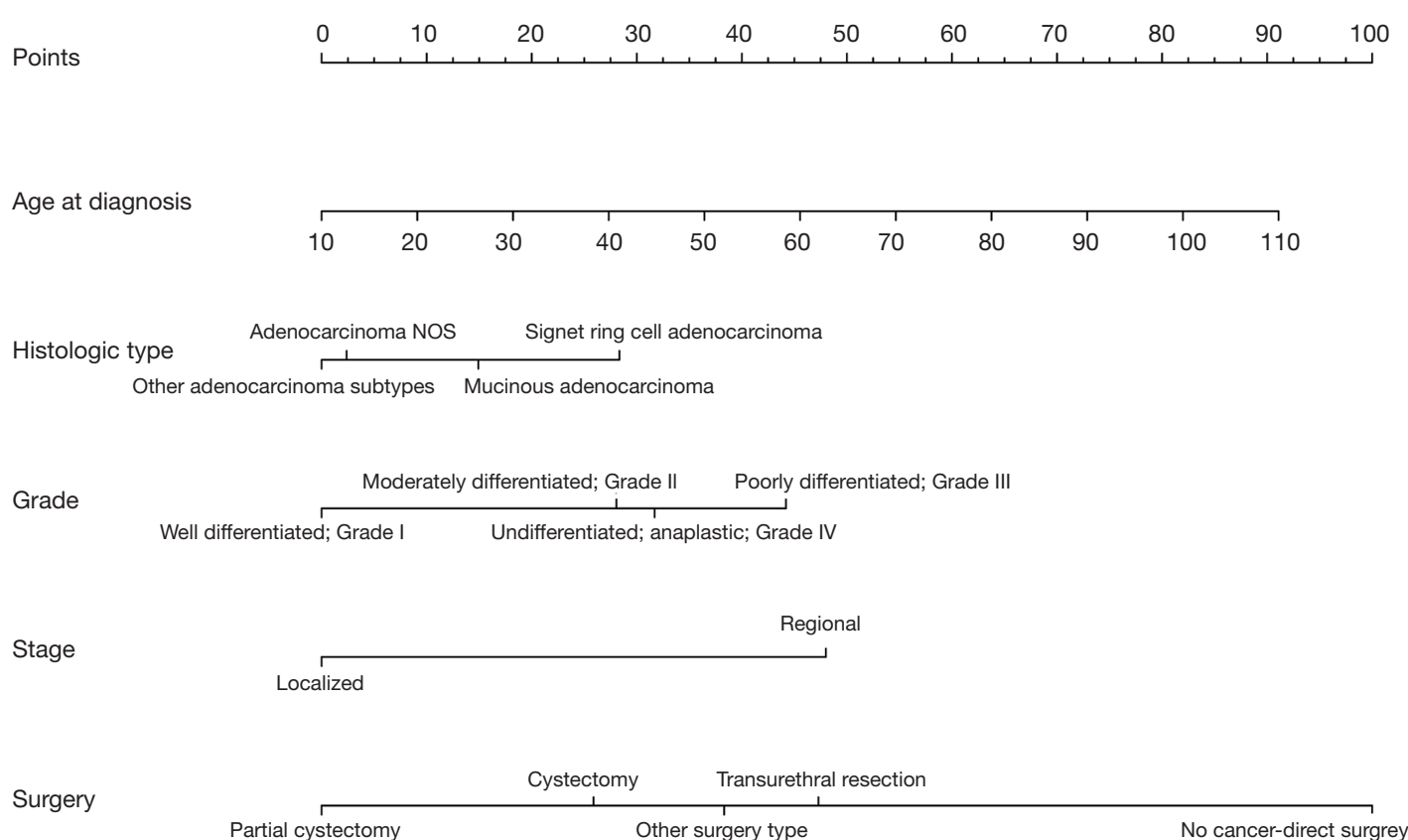

Total points

\begin{tabular}{|c|c|c|c|c|}
\hline 50 & 100 & 150 & 200 & 250 \\
\hline
\end{tabular}

Linear predictor

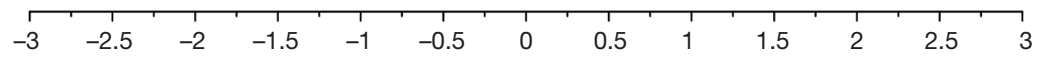

1-year survival probability

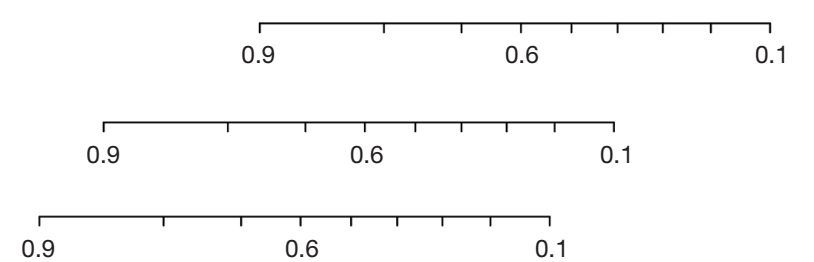

3-year survival probability

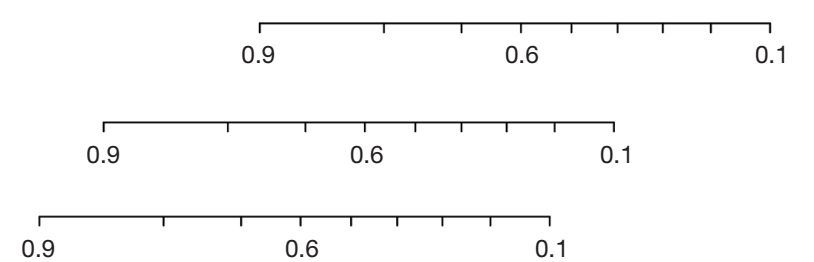

5-year survival probability

Figure 2 Nomogram predicting 1-, 3- and 5-year CSS of patients with primary adenocarcinoma of the bladder. CSS, cancer-specific survival.

Harrel's C indices for CSS were 0.729 (95\% CI, 0.707$0.751)$ in the training cohort and 0.716 (95\% CI, $0.678-$ $0.754)$ in the validation cohort. The AUC values were 0.769 , 0.735 and 0.724 for 1,3 and 5 -year CSS in the training cohort, and $0.738,0.727$ and 0.713 for 1,3 and 5 -year in the validation cohort, respectively. These values indicated that the nomogram had good discriminative capability, as shown in Figure 3. Calibration plots indicated that the predicted 1, 3 and 5 -year CSS rates were close to actual observations, as shown in Figure 4.

\section{Discussion}

Primary adenocarcinoma of the urinary bladder (ACB) is a rare malignant tumor with a wide range of clinical outcomes. Accurate prediction of disease prognosis plays an important role in disease management. Nomograms are considered reliable visualization models and have been widely used to predict individual risks of particular events, such as metastatic progression, survival and recurrence etc. (10).

In the present study, using data from a large, nationwide, population-based database, independent prognostic factors 

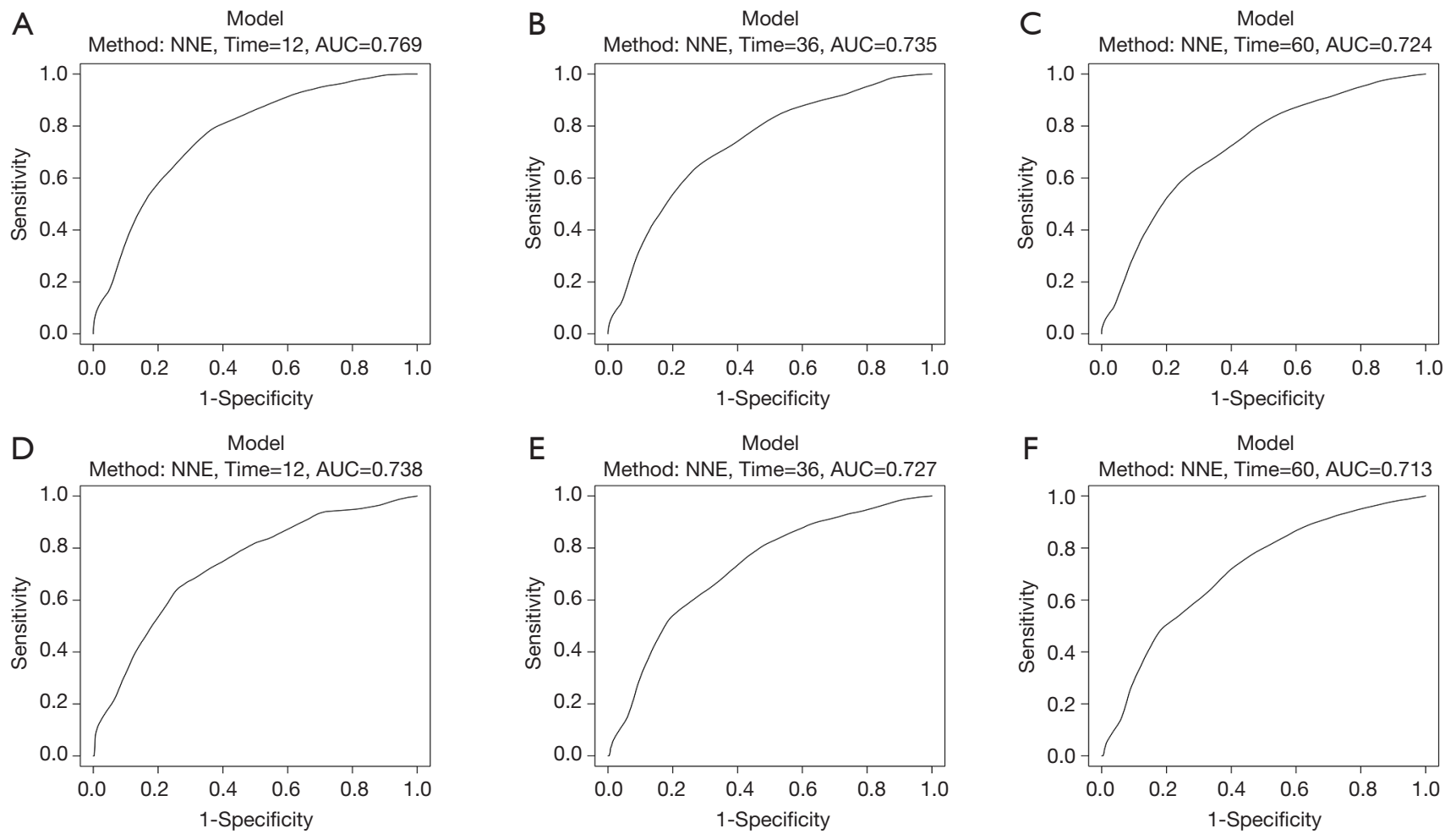

Figure 3 ROC curves. The ability of the Nomogram to be measured by the AUC. (A) 1-year, (B) 3-year and (C) 5-year ROC curves in the training cohort; (D) 1-year, (E) 3-year and (F) 5-year ROC curves in the validation cohort. ROC, receiver operating characteristics curve; AUC, the area under the receiver operating characteristics curve.

were identified for patients with NMACB and thus a nomogram was established to predict personalized CSS. The nomogram used the following prognostic factors previously demonstrated to be associated with survival of ACB patients: age at diagnosis (16), histological type $(17,18)$, grade $(19)$, stage $(16,19,20)$ and surgery $(17,19)$.

Using these independent prognostic factors, we constructed a nomogram that combined treatment with particular clinical parameters. The nomogram demonstrated that age and surgery are the most important factors affecting prognosis, while histological subtypes have limited influence. One interesting finding was that patients with undifferentiated or anaplastic tumors had better survival outcomes than those with a poorly differentiated tumor grade.

Wright et al. (21) and Natale et al. (17) demonstrated that compared with patients with non-urachal tumors, individuals with urachal adenocarcinoma had better CSS, even if patients with urachal adenocarcinoma were more likely to have a higher tumor stage than those with non- urachal adenocarcinoma. The reported 5-year cancer specific survival rates were $48 \%$ and $35 \%$ for urachal and non-urachal adenocarcinoma, respectively (21). In the present study, primary tumor site was found not to be an independent prognostic factor. This inconsistency may be due to our focus on non-metastatic patients. An additional analysis was later performed that included metastatic patients, the results of which matched those observed in earlier studies (data not presented in the article) $(17,21)$.

Surgery with or without adjuvant radiation or chemotherapy is the principal form of treatment for patients with ACB. The most commonly used surgical procedure is radical or partial cystectomy with or without node dissection (22). The role of chemotherapy and radiotherapy in primary adenocarcinoma of the bladder is controversial. No specific recommendations exist for the use or type of chemotherapy or radiotherapy $(4,23)$. Szarvas et al. (24) and Tatli et al. (25) believed that chemotherapy regimens containing 5-FU can improve the prognosis of ACB, while others have indicated that radiotherapy, or neo-adjuvant 

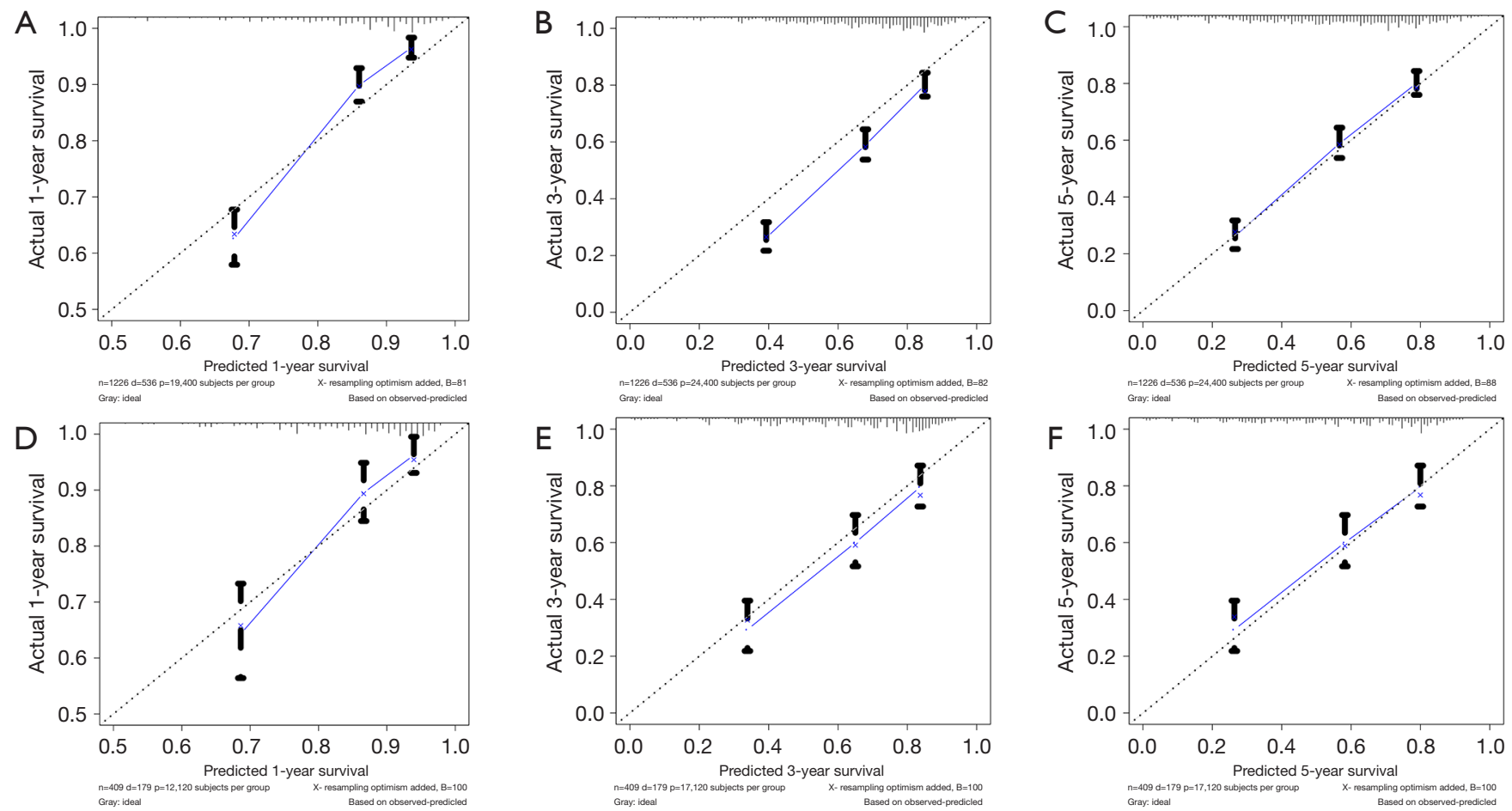

Figure 4 CSS calibration plots. The relationship between the predicted survival probabilities and actual values. (A) 1-year, (B) 3-year and (C) 5-year calibration plots in the training cohort; (D) 1-year, (E) 3-year and (F) 5-year calibration plots in the validation cohort. CSS, cancer-specific survival.

or adjuvant chemotherapy have not proved efficacious in adenocarcinoma of the bladder $(22,26)$. Conversely, in the present study, chemotherapy or radiotherapy were found not to be independent prognostic factors.

Previous studies have shown that ACB patients have a higher risk of suffering from non-organ-confined disease (3). In this study, 1,156 (70.70\%) patients had regional tumor stage. This may due to its intramural growth leading to late-onset hematuria, urinary tract irritation, or other symptoms, leading to a late stage when diagnosed (27).

Generally speaking, the TNM staging system is strongly related to survival outcomes. However, different survival outcomes were also observed among patients at the same stage. These differences may because of the lack of other prognostic factors such as age and grade, etc. For this reason, a more accurate method of predicting individualized survival outcomes in ACB patients is required and a nomogram is a suitable method for this purpose. In the present study, we developed a nomogram capable of predicting CSS in ACB. The AUC values for 1, 3, and 5 -year CSS indicated good discriminative capability of the nomogram. Calibration plots indicated that the predicted 1,3 , and 5 -year CSS rates were close to actual observations, confirming the validity and reliability of the nomogram.

We should note that there are a number of limitations to this study. Firstly, our research was retrospective which inevitably resulted in selection bias. Secondly, the distinction between urachal and non-urachal primary adenocarcinoma has always been difficult to ascertain, both in clinic and by pathology $(20,28,29)$, resulting in potential misclassification bias. Consequently, we performed the analysis again after excluding the primary site of dome lesion. There was no significant change to the results (data not shown in the article). Thirdly, the SEER database lacks information about treatment strategies, family history, occupation, comorbid conditions, tumor markers or biochemical or immunological factors, which may cause confounding or selection bias. Fourthly, we used the SEER historic stage A (localized, regional, distant and unstaged) classification, which is not widely accepted, because it is the only staging system used continuously throughout the study period from 1973 to 2015 . A nomogram based on the TNM staging system was also constructed (not shown in the article), but it did not significantly improve the performance of the prediction. Lastly, but not least, external validation and prospective clinical trials are essential to establish the 
accuracy and clinical utility of the models. However, this is a real-world study based on a large sample, and these limitations do not weaken our conclusions.

\section{Conclusions}

In summary, age, grade, stage, histologic type, and surgery were found to be independent prognostic factors for CSS. We developed an effective nomogram for predicting 1, 3 and 5-year CSS of NMACB patients, which can help clinicians accurately predict risk of mortality and allow them to propose a personalized treatment modality.

\section{Acknowledgments}

The authors are grateful to all the staff at the National Cancer Institute (USA) for their contribution to the SEER program.

Funding: None.

\section{Footnote}

Reporting Checklist: The authors have completed the STROBE reporting checklist. Available at http://dx.doi. org/10.21037/tcr-20-354

Peer Review File: Available at http://dx.doi.org/10.21037/tcr20-354

Conflicts of Interest: All authors have completed the ICMJE uniform disclosure form (available at http://dx.doi. org/10.21037/tcr-20-354). The authors have no conflicts of interest to declare.

Ethical Statement: The authors are accountable for all aspects of the work in ensuring that questions related to the accuracy or integrity of any part of the work are appropriately investigated and resolved. This study was conducted in accordance with standard guidelines and was approved by the local Ethics Committee. We also got permission from the National Cancer Institute USA to access the SEER dataset for research purposes only (reference number: 18015-Nov2017). All the data from the SEER database were de-identified, and the extracted data did not require informed consent.

Open Access Statement: This is an Open Access article distributed in accordance with the Creative Commons
Attribution-NonCommercial-NoDerivs 4.0 International License (CC BY-NC-ND 4.0), which permits the noncommercial replication and distribution of the article with the strict proviso that no changes or edits are made and the original work is properly cited (including links to both the formal publication through the relevant DOI and the license). See: https://creativecommons.org/licenses/by-nc-nd/4.0/.

\section{References}

1. Hurst C, Rosenberg J, Knowles M. SnapShot: Bladder Cancer. Cancer Cell 2018;34:350-350.e1.

2. Hamilou Z, North S, Canil C, et al. Management of urachal cancer: A review by the Canadian Urological Association and Genitourinary Medical Oncologists of Canada. Can Urol Assoc J 2020;14:E57-64.

3. Yu B, Zhou J, Cai H, et al. Neoadjuvant chemotherapy for primary adenocarcinomas of the urinary bladder: a singlesite experience. BMC Urol 2015;15:3.

4. Alanee S, Alvarado-Cabrero I, Murugan P, et al. Update of the International Consultation on Urological Diseases on bladder cancer 2018: non-urothelial cancers of the urinary bladder. World J Urol 2019;37:107-14.

5. Antoni S, Ferlay J, Soerjomataram I, et al. Bladder Cancer Incidence and Mortality: A Global Overview and Recent Trends. Eur Urol 2017;71:96-108.

6. el-Mekresh MM, el-Baz MA, Abol-Enein H, et al. Primary adenocarcinoma of the urinary bladder: a report of 185 cases. Br J Urol 1998;82:206-12.

7. Cancer Genome Atlas Research Network. Comprehensive molecular characterization of urothelial bladder carcinoma. Nature 2014;507:315-22.

8. Babjuk M, Burger M, Zigeuner R, et al. EAU Guidelines on Non-Muscle-invasive Urothelial Carcinoma of the Bladder: Update 2013. Eur Urol 2013;64:639-53.

9. Burger M, Catto JWF, Dalbagni G, et al. Epidemiology and Risk Factors of Urothelial Bladder Cancer. Eur Urol 2013;63:234-41.

10. Collins GS, Reitsma JB, Altman DG, Moons KG. Transparent reporting of a multivariable prediction model for individual prognosis or diagnosis (TRIPOD): the TRIPOD statement. BMJ 2015;350:g7594.

11. Wang SJ, Lemieux A, Kalpathy-Cramer J, et al. Nomogram for predicting the benefit of adjuvant chemoradiotherapy for resected gallbladder cancer. J Clin Oncol 2011;29:4627-32.

12. Cao J, Yuan P, Wang L, et al. Clinical Nomogram for Predicting Survival of Esophageal Cancer Patients after 
Esophagectomy. Sci Rep 2016;6:26684.

13. Wu S, Zheng J, Li Y, et al. A Radiomics Nomogram for the Preoperative Prediction of Lymph Node Metastasis in Bladder Cancer. Clin Cancer Res 2017;23:6904-11.

14. Zhang Y, Hong YK, Zhuang DW, et al. Bladder cancer survival nomogram. Medicine (Baltimore) 2019;98:e17725.

15. Tang F, He Z, Lu Z, et al. Application of nomograms in the prediction of overall survival and cancer-specific survival in patients with T1 high-grade bladder cancer. Exp Ther Med 2019;18:3405-14.

16. Pinthus JH, Haddad R, Trachtenberg J, et al. Population Based Survival Data on Urachal Tumors. J Urol 2006;175:2042-7.

17. Natale C, Leinwand GZ, Chiang J, et al. Reviewing the Demographic, Prognostic, and Treatment Factors of Primary Adenocarcinoma of the Bladder: A SEER Population-based Study. Clin Genitourin Cancer 2019;17:380-8.

18. Wang J, Wang FW, Kessinger A. The impact of signetring cell carcinoma histology on bladder cancer outcome. World J Urol 2012;30:777-83.

19. Mylonas KS, O Malley P, Ziogas IA, et al. Malignant urachal neoplasms: A population-based study and systematic review of literature. Urol Oncol 2017;35:33. e11-e19.

20. Gopalan A, Sharp DS, Fine SW, et al. Urachal Carcinoma A Clinicopathologic Analysis of 24 Cases With Outcome
Correlation. Am J Surg Pathol 2009;33:659-68.

21. Wright JL, Porter MP, Li CI, et al. Differences in survival among patients with urachal and nonurachal adenocarcinomas of the bladder. Cancer 2006;107:721-8.

22. Porten SP, Willis D, Kamat AM. Variant histology: role in management and prognosis of nonmuscle invasive bladder cancer. Curr Opin Urol 2014;24:517-23.

23. Tsironis G, Bamias A. Treating bladder adenocarcinoma. Transl Androl Urol 2018;7:S699-S701.

24. Szarvas T, Modos O, Niedworok C, et al. Clinical, prognostic, and therapeutic aspects of urachal carcinoma-A comprehensive review with meta-analysis of 1,010 cases. Urol Oncol 2016;34:388-98.

25. Tatli AM, Uysal M, Goksu SS, et al. Complete response of primary bladder adenocarcinoma with the FOLFOX4 regimen. Urol Int 2015;94:363-5.

26. National Comprehensive Cancer Network. Bladder cancer, version 2, Available online: https://www.nccn.org/ professionals/physician_gls/pdf/bladder.pdf2017

27. Henly DR, Farrow GM, Zincke H. Urachal cancer: role of conservative surgery. Urology 1993;42:635-9.

28. Johnson DE, Hodge GB, Abdul-Karim FW, et al. Urachal carcinoma. Urology 1985;26:218-21.

29. Maslov DV, Thomas K, Matrana M. Chemotherapy Toxicity Confirms Diagnosis of Urachal Carcinoma. Clin Genitourin Cancer 2019;17:e913-5.
Cite this article as: Yu DD, Dong $\mathrm{H}$, Chen WK, Chen $\mathrm{K}$, Wu ZG, Li CD, Cai J, Xiao YB. Development and validation of a nomogram for predicting survival in patients with nonmetastatic primary adenocarcinoma of the bladder. Transl Cancer Res 2020;9(9):5155-5165. doi: 10.21037/tcr-20-354 